\title{
Synthesis, Characterization and Evaluation of Anti-Inflammatory and Analgesic Activity of Some Novel Quinoline Based Thiazolidinone Heterocycles
}

\author{
Atef M. Amer ${ }^{*}$, Ali Deeb1, Wafaa I. El-Eraky², Sally A. El Awdan² and Sebaey \\ Mahgoub $^{3}$ \\ ${ }^{1}$ Department of Chemistry, Faculty of Science, Zagazig University, Egypt. \\ ${ }^{2}$ Department of Pharmacology, National Research Centre, Cairo, Egypt. \\ ${ }^{3}$ Research and Development, Unipharma, El-Obour City, Egypt.
}

\begin{abstract}
$\mathrm{N}$ this paper we report the synthesis of some quinoline based thiazolidinone derivatives (13-22) in a three-step process. Condensation of 2-hydrazinylquinoline $\mathbf{2}$ with different aromatic aldehydes gave the corresponding Schiff bases 3-10 which in turn were reacted with thioglycolic acid to furnish the corresponding thiazolidinone derivatives 13-20. Reaction of compound 2 with isatin or methyl isatin gave 1-sustituted-3-(2-(quinolin-2-yl)hydrazono) indolin-2-ones $\mathbf{1 1}$ and 12, which were converted to 1-substituted 3'-(quinolin-2-ylamino) spiro[indoline-3,2'-thiazolidine]-2,4'-dione $\mathbf{2 1}$ and $\mathbf{2 2}$ by cyclocondensation with thioglycolic acid. All newly synthesized compounds have been characterized by means of elemental analyses, IR, ${ }^{1} \mathrm{H}$ NMR and MS. Furthermore, all new thiazolidinone derivatives were evaluated for their anti-inflammatory and analgesic activity. The Study results revealed that the highest anti-inflammatory potency was gained by 6 derivatives according to the following order $\mathbf{2 2}>\mathbf{1 7}>\mathbf{1 3}>\mathbf{1 4}>\mathbf{2 1}>\mathbf{1 5}$, showing a good edema inhibition compared to the reference drug indomethacin. Compound $\mathbf{2 2}$ carrying indole ring system inhibited the edema volume significantly at the $1^{\text {st }} \mathrm{h}$ post administration, and the activity was enhanced up to the $4^{\text {th }} \mathrm{h}$ giving a promising edema volume inhibition compared to that produced by indomethacin. The longest duration of analgesic action up to 90 min post compounds administration was obtained by the compounds $\mathbf{1 3}, 17$ and $\mathbf{2 2}$, they exhibited potent analgesia compared to that obtained by aspirin.
\end{abstract}

Keywords: Quinoline, Thiazolidinone, spiro[indoline-3,2'-thiazolidine]-2,4'-dione, Synthesis, Anti-inflammatory and analgesic activity.

\section{Introduction}

The chemistry of quinoline derivatives has been of increasing interest due to their vast chemical reactivity, biological and pharmaceutical activities [1-5]. It has been reported that these derivatives possesses anti-tuberculosis [6,7], Antiplasmodial [4], Antibacterial [5], antihistamine [6], anticancer [11-14], anti-hypertensive [9], antifungal [10], antimalarial [11], anti-HIV [12] and antioxidant activities [13]. On the other hand, thiazole and thiazolidinone derivatives are known to exhibit diverse biological activities[14-16], they have anti-inflammatory $[20,21]$, anticancer, antimicrobial [19], anti-tuberculosis [20] and antioxidant activity [21]. Inflammation is a fundamental physiological process that is not only essential for survival but also at the same time is one of the major causes of human mortality $[25,26]$. It's known that, non-steroidal anti-inflammatory drugs (NSAIDs) are used to treat a wide variety of illnesses and diseases, such as inflammation [24], cancers [25] and the peripheral and central nervous system diseases [26]. The anti-inflammatory effect of NSAIDs arises from their ability to inhibit both $\mathrm{COX}-1$ and COX-2 isoforms of cyclooxygenase (COX) enzyme[27]. Literature survey revealed that some of the synthesized derivatives having quinoline ring or bearing thiazole ring have significant antiinflammatory and analgesic activity [31-33, 20, 21]. In the light of above mentioned facts and our interest in designing new biologically active molecules, our efforts were directed towards

*Corresponding author e-mail: atefamer55@yahoo.com

DOI: 10.21608/EJCHEM.2018.5155.1456

C2017 National Information and Documentation Center (NIDOC) 
the synthesis of new heterocyclic compounds containing quinoline moiety with anticipated biological activities. In this paper, we report synthesis of novel quinoline based thiazolidinone derivatives as anti-inflammatory and analgesic agents.

\section{Material and Methods}

General

All melting points were determined in openglass capillaries and are uncorrected. IR spectra were recorded on a BRUKER Vector 22 Germany spectrometer (KBr). ${ }^{1} \mathrm{H}$ NMR spectra were recorded on (Bruker) $400 \mathrm{MHz}$ spectrometer using TMS as an internal reference. The Electron Impact mass spectra were obtained at $70 \mathrm{eV}$ using Shimadzu QP-2010 Plus mass spectrometer. The reactions were followed up by thin-layer chromatography (TLC) on silica gel F254 aluminum sheets (Merck), and the spots were detected by UV lamp at 254-365 $\mathrm{nm}$.

\section{Preparation of 2-hydrazinylquinoline (2)}

2-Chloroquinoline 1 (1.0 g, $6.1 \mathrm{mmol})$ and hydrazine monohydrate $(3 \mathrm{~mL})$ in n-butanol $(10$ $\mathrm{ml}$ ) were refluxed for $6 \mathrm{~h}$. The solvent was removed under reduced pressure giving a brownish orange residue, the residue was triturated with ethanol then filtered to give compound $\mathbf{2}$, orange crystals, yield $86.5 \%$; mp $140-142{ }^{\circ} \mathrm{C}$; IR (KBr, cm-1): 3282, $3188(\mathrm{NH}), 3042\left(\mathrm{CH}_{\text {arom }}\right), 1621(\mathrm{C}=\mathrm{N})$.

General procedure for the synthesis of (E)-2-(2arylidenehydrazinyl) quinoline (3-9)

A mixture of compound $2(1.0 \mathrm{~g}, 6.3 \mathrm{mmol})$ and the substituted aldehydes $(6.3 \mathrm{mmol})$, were refluxed in ethanol $(20 \mathrm{~mL})$ containing few drops of glacial acetic acid for $3 \mathrm{~h}$. The solvent was reduced to its half, and allowed to cool. The separated solid was filtered, dried, and recrystallized from ethanol.

(E)-2-(2-(4-chlorobenzylidene)hydrazinyl) quinoline (3)

Pale yellow solid; yield 85.5\%; mp 123-125 ${ }^{\circ} \mathrm{C}$; IR $\left(\mathrm{KBr}, \mathrm{cm}^{-1}\right): 3429(\mathrm{NH}), 3045\left(\mathrm{CH}_{\text {arom }}\right), 1621$ $(\mathrm{CH}=\mathrm{N}) ; \mathrm{MS}(\mathrm{m} / \mathrm{z}): 281\left[\mathrm{M}^{+}, 0.21 \%\right], 247\left[\mathrm{M}^{+}-\right.$ $\mathrm{Cl}, 0.15 \%$ ], 171 [ $\mathrm{M}^{+}-\mathrm{PhCl}, 7.24 \%$ ], 69 [base peak, $100 \%$ ]; ${ }^{1} \mathrm{H}$ NMR $\left(\mathrm{DMSO}_{-}\right)$: $\delta(\mathrm{ppm})=$ 13.3 (s, 1H, NH), 8.6 (s, 1H, $\mathrm{CH}=\mathrm{N}), 8.13-7.36$ (m, 10H, Ar-H). Anal. Calcd for $\mathrm{C}_{16} \mathrm{H}_{12} \mathrm{ClN}_{3}$ (281.74): C, 68.21; H, 4.29; N, 14.91. Found: C, $68.06 ; \mathrm{H}, 4.13 ; \mathrm{N}, 14.75$.

\section{(E)-2-(2-(4-methoxybenzylidene)hydrazinyl)}

quinoline (4)

Yellow solid; yield $86 \%$; mp $155-157{ }^{\circ} \mathrm{C}$; IR (KBr, cm-1): $3431(\mathrm{NH}), 3106\left(\mathrm{CH}_{\text {arom }}\right), 2966$ $\left(\mathrm{CH}_{\text {alinh }}\right), 1599(\mathrm{CH}=\mathrm{N}), 1023\left(\mathrm{OCH}_{3}\right) ; \mathrm{MS}(\mathrm{m} / \mathrm{z})$ : $277\left[\mathrm{M}^{+}, 3.33 \%\right], 247\left[\mathrm{M}^{+}-\mathrm{OCH}_{3}, 1.05 \%\right], 69$ [base peak, $100 \%$ ]; ${ }^{1} \mathrm{H}$ NMR (DMSO-d $\left.{ }_{6}\right): \delta(\mathrm{ppm})$ $=11.2(\mathrm{~s}, 1 \mathrm{H}, \mathrm{NH}), 8.6(\mathrm{~s}, 1 \mathrm{H}, \mathrm{CH}=\mathrm{N}), 7.82-7.04$ $(\mathrm{m}, 10 \mathrm{H}, \mathrm{Ar}-\mathrm{H}), 3.8\left(\mathrm{~s}, 3 \mathrm{H}, \mathrm{OCH}_{3}\right)$. Anal. Calcd for $\mathrm{C}_{17} \mathrm{H}_{15} \mathrm{~N}_{3} \mathrm{O}(277.33)$ : $\mathrm{C}, 73.63 ; \mathrm{H}, 5.45 ; \mathrm{N}$, 15.15. Found: C, 73.50; H, 5.32; N, 15.02.

(E)-N,N-dimethyl-4-((2-(quinolin-2-yl) hydrazono)methyl)aniline (5) [30]

Yellow solid, yield $65.5 \%$; mp $260-262{ }^{\circ} \mathrm{C}$; IR (KBr, cm $\left.{ }^{-1}\right): 3428(\mathrm{NH}), 3045\left(\mathrm{CH}_{\text {arom }}\right), 2911$ $\left(\mathrm{CH}_{\text {aliph }}\right), 1651(\mathrm{CH}=\mathrm{N}) ; \mathrm{MS}(\mathrm{m} / \mathrm{z}): 290\left[\mathrm{M}^{+}\right.$, $0.85 \%], 262\left[\mathrm{M}^{+}-2 \mathrm{CH}_{3}, 0.65 \%\right], 247\left[\mathrm{M}^{+}-\right.$ $\mathrm{N}\left(\mathrm{CH}_{3}\right) 2,0.36 \%$ ], 170 [base peak, $100 \%$ ]; ${ }^{1} \mathrm{H}$ NMR $\left.(\text { DMSO-d })_{6}\right): \delta(\mathrm{ppm})=13.2(\mathrm{~s}, 1 \mathrm{H}, \mathrm{NH})$, $8.55(\mathrm{~s}, 1 \mathrm{H}, \mathrm{CH}=\mathrm{N}), 8.20-7.45(\mathrm{~m}, 10 \mathrm{H}, \mathrm{Ar}-$ $\mathrm{H}), 3.02\left(\mathrm{~s}, 6 \mathrm{H}, 2 \mathrm{CH}_{3}\right)$. Anal. Calcd for $\mathrm{C}_{18} \mathrm{H}_{18} \mathrm{~N}_{4}$ (290.37): C, 74.46; H, 6.25; N, 19.30. Found: C, $74.31 ; \mathrm{H}, 6.09 ; \mathrm{N}, 19.14$.

(E)-2-(2-(2-chlorobenzylidene)hydrazinyl) quinoline (6)

Yellow solid; yield $77 \%$; mp 278-280 ${ }^{\circ} \mathrm{C}$; IR (KBr, cm $\left.{ }^{-1}\right): 3393(\mathrm{NH}), 3063\left(\mathrm{CH}_{\text {arom }}\right), 1647$ $(\mathrm{CH}=\mathrm{N}) ; \mathrm{MS}(\mathrm{m} / \mathrm{z}): 281\left[\mathrm{M}^{+}, 0.34 \%\right], 247\left[\mathrm{M}^{+}-\right.$ $\mathrm{Cl}, 1.05 \%], 171$ [M+-PhCl, $8.73 \%$ ], 69 [base peak, $100 \%$ ]; ${ }^{1} \mathrm{H}$ NMR $\left(\right.$ DMSO-d $\left._{6}\right): \delta(\mathrm{ppm})=$ $13.6(\mathrm{~s}, 1 \mathrm{H}, \mathrm{NH}), 8.9(\mathrm{~s}, 1 \mathrm{H}, \mathrm{CH}=\mathrm{N}), 8.83-7.46$ (m, 10H, Ar-H). Anal. Calcd for $\mathrm{C}_{16} \mathrm{H}_{12} \mathrm{ClN}_{3}$ (281.74): C, 68.21; H, 4.29; N, 14.91. Found: C, $68.06 ; \mathrm{H}, 4.13 ; \mathrm{N}, 14.75$.

(E)-2-(2-benzylidenehydrazinyl) quinoline (7)

Yellow solid; yield $73.5 \%$; mp $105-107{ }^{\circ} \mathrm{C}$; IR $\left(\mathrm{KBr}, \mathrm{cm}^{-1}\right): 3425(\mathrm{NH}), 3046\left(\mathrm{CH}_{\text {arom }}\right), 1663$, $(\mathrm{CH}=\mathrm{N}) ; \mathrm{MS}(\mathrm{m} / \mathrm{z}): 247\left[\mathrm{M}^{+}, 1.05 \%\right], 171\left[\mathrm{M}^{+}-\right.$ $\mathrm{Ph}, 8.73 \%$ ], 69 [base peak, $100 \%$ ]; ${ }^{1} \mathrm{H}$ NMR $\left(\mathrm{DMSO}_{6}\right): \delta(\mathrm{ppm})=11.2(\mathrm{~s}, 1 \mathrm{H}, \mathrm{NH}), 8.72$ (s, 1H, CH=N), $7.8-7.35(\mathrm{~m}, 11 \mathrm{H}$, Ar-H). Anal. Calcd for $\mathrm{C}_{16} \mathrm{H}_{13} \mathrm{~N}_{3}$ (247.30): C, 77.71; H, 5.30; N, 16.99. Found: C, 77.56; H, 5.16; N, 16.84 .

(E)-2-((2-(quinolin-2-yl)hydrazono)methyl) phenol ( 8$)$

Yellow solid; yield $79 \%$; mp 220-222 ${ }^{\circ} \mathrm{C}$; IR $\left(\mathrm{KBr}, \mathrm{cm}^{-1}\right): 3752(\mathrm{OH}), 3425(\mathrm{NH}), 3043$ $\left(\mathrm{CH}_{\text {arom }}\right), 2916\left(\mathrm{CH}_{\text {aliph }}\right), 1621(\mathrm{CH}=\mathrm{N}) ; \mathrm{MS}(\mathrm{m} / \mathrm{z})$ : $263\left[\mathrm{M}^{+}, 0.44 \%\right], 247\left[\mathrm{M}^{+}-\mathrm{OH}, 2.23 \%\right] ;{ }^{1} \mathrm{H}$ NMR (DMSO-d $\left.)_{6}\right): \delta(\mathrm{ppm})=13.2(\mathrm{~s}, 1 \mathrm{H}, \mathrm{NH})$, $11.2(\mathrm{~s}, 1 \mathrm{H}, \mathrm{OH}), 9.0(\mathrm{~s}, 1 \mathrm{H}, \mathrm{CH}=\mathrm{N}), 7.70-$ 6.9 (m, 10H, Ar-H). Anal. Calcd for $\mathrm{C}_{16} \mathrm{H}_{13} \mathrm{~N}_{3} \mathrm{O}$ (263.30): C, 72.99; H, 4.98; N, 15.96. Found: C, 
72.84; H, 4.82; N, 15.80 .

(E)-2-(2-(3-nitrobenzylidene)hydrazinyl) quinoline (9)

Yellow solid; yield $79 \%$; mp $132-134{ }^{\circ} \mathrm{C}$; IR (KBr, cm $\left.{ }^{-1}\right): 3426(\mathrm{NH}), 3046\left(\mathrm{CH}_{\text {arom }}\right), 1617$ $(\mathrm{CH}=\mathrm{N}), 1518,1347\left(\mathrm{NO}_{2}\right) ; \mathrm{MS}(\mathrm{m} / \mathrm{z}): 292[\mathrm{M}+$, $27.69 \%$ ], $247\left[\mathrm{M}^{+}-\mathrm{NO}_{2}, 0.06 \%\right.$ ], 170 [base peak , $100 \%$ ]; ${ }^{1} \mathrm{H}$ NMR $\left(\right.$ DMSO-d $\left._{6}\right): \delta(\mathrm{ppm})=11.8(\mathrm{~s}$, $1 \mathrm{H}, \mathrm{NH}), 8.49(\mathrm{~s}, 1 \mathrm{H}, \mathrm{CH}=\mathrm{N}), 8.22-7.29(\mathrm{~m}$, $10 \mathrm{H}, \mathrm{Ar}-\mathrm{H})$. Anal. Calcd for $\mathrm{C}_{16} \mathrm{H}_{12} \mathrm{~N}_{4} \mathrm{O}_{2}$ (292.30): C, 65.75; H, 4.14; N, 19.17. Found: C, 65.60; H, $3.99 ; \mathrm{N}, 19.01$.

Synthesis of (E)-2-(2-(naphthalen-1-ylmethylene) hydrazinyl)quinoline (10)

To a solution of compound $2(1.0 \mathrm{~g}, 6.3 \mathrm{mmol})$ and 1-naphthaldehyde $(6.3 \mathrm{mmol})$, in ethanol $(20 \mathrm{~mL})$ few drops of glacial acetic acid were added, and the mixture was refluxed for $3 \mathrm{~h}$. The solvent was reduced to its half, and allowed to cool. The separated solid was filtered, dried, and recrystallized from ethanol.

Yellow solid; yield $82 \%$; mp 280-282 ${ }^{\circ} \mathrm{C}$; IR ( $\left.\mathrm{KBr}, \mathrm{cm}^{-1}\right): 3426(\mathrm{NH}), 3057\left(\mathrm{CH}_{\text {arom }}\right), 1644$ $(\mathrm{CH}=\mathrm{N})$; MS (m/z): $297\left[\mathrm{M}^{+} 0.15 \%\right.$ ], 259 [base peak, 100]; ${ }^{1} \mathrm{H}$ NMR $\left(\right.$ DMSO-d $\left._{6}\right): \delta(\mathrm{ppm})=9.54$ (s, 1H, NH), 8.54 (s, 1H, CH=N), $8.20-7.49$ (m, $13 \mathrm{H}, \mathrm{Ar}-\mathrm{H})$. Anal. Calcd for $\mathrm{C}_{20} \mathrm{H}_{15} \mathrm{~N}_{3}$ (297.36): C, 80.78; H, 5.08; N, 14.13. Found: C, 80.63; H, $4.92 ; \mathrm{N}, 13.98$.

General procedure for the synthesis of (Z)-1sustituted-3-(2-(quinolin-2-yl)hydrazono)indolin2-one (11) and (12)

A mixture of compound $2(1.0 \mathrm{~g}, 6.3 \mathrm{mmol})$ and isatin or methyl isatin $(6.3 \mathrm{mmol})$, was refluxed in ethanol $(20 \mathrm{~mL})$ containing few drops of glacial acetic acid for $3 \mathrm{~h}$. The solvent was reduced to its half, and allowed to cool. The separated solid was filtered, dried, and recrystallized from ethanol.

(Z)-3-(2-(quinolin-2-yl)hydrazono)indolin-2on (11) [31]

Pale orange solid; yield $81 \%$; $\mathrm{mp} 278-280{ }^{\circ} \mathrm{C}$; IR ( $\left.\mathrm{KBr}, \mathrm{cm}^{-1}\right): 3431(\mathrm{NH}), 3090\left(\mathrm{CH}_{\text {arom }}\right), 1646$ $(\mathrm{C}=\mathrm{O}), 1616(\mathrm{CH}=\mathrm{N}) ; \mathrm{MS}(\mathrm{m} / \mathrm{z}): 288\left[\mathrm{M}^{+}, 9.01\right.$ $\%$ ], $262\left[\mathrm{M}^{+}-\mathrm{CO}, 0.35 \%\right.$ ], 259 [base peak, 100 \%]; ${ }^{1} \mathrm{H}$ NMR $\left(\mathrm{DMSO}_{6}\right): \delta(\mathrm{ppm})=13.2(\mathrm{~s}, 1 \mathrm{H}$, $\mathrm{NH}), 11.2$ (s, 1H, NH, indole), 7.99- 6.79 (m, $10 \mathrm{H}, \mathrm{Ar}-\mathrm{H})$. Anal. Calcd for $\mathrm{C}_{17} \mathrm{H}_{12} \mathrm{~N}_{4} \mathrm{O}$ (288.31): C, 70.82; H, 4.20; N, 19.43 Found: C, 70.68; H, $4.04 ; \mathrm{N}, 19.29$.

(Z)-1-methyl-3-(2-(quinolin-2-yl)hydrazono) indolin-2-one (12)

Orange solid; yield $77.5 \%$; mp $190-192{ }^{\circ} \mathrm{C}$; IR $\left(\mathrm{KBr}, \mathrm{cm}^{-1}\right): 3430(\mathrm{NH}), 3051\left(\mathrm{CH}_{\text {arom }}\right), 2927$ $\left(\mathrm{CH}_{\text {aliph }}\right), 1674(\mathrm{C}=\mathrm{O}), 1606(\mathrm{CH}=\mathrm{N}) ; \mathrm{MS}(\mathrm{m} / \mathrm{z})$ : $302\left[\mathrm{M}^{+}, 0.3 \%\right], 288\left[\mathrm{M}^{+}-\mathrm{CH} 3,8.86 \%\right], 262$ $\left[\mathrm{M}^{+}-\mathrm{CH}_{3}\right.$ and $\mathrm{CO}, 0.67 \%$ ], 69 [base peak, 100 \%]; ${ }^{1} \mathrm{H}$ NMR $\left(\right.$ DMSO-d $\left._{6}\right): \delta(\mathrm{ppm})=12.97(\mathrm{~s}$, $1 \mathrm{H}, \mathrm{NH}), 8.43-7.16(\mathrm{~m}, 10 \mathrm{H}, \mathrm{Ar}-\mathrm{H}), 3.31$ (s, $3 \mathrm{H}, \mathrm{CH}_{3}$ ). Anal. Calcd for $\mathrm{C}_{18} \mathrm{H}_{14} \mathrm{~N}_{4} \mathrm{O}$ (302.34): C, 71.51; H, 4.67; N, 18.53. Found: C, 71.36; H, $4.52 ; \mathrm{N}, 18.40$.

General procedures for the synthesis of 2-substituted-3-(quinolin-2-ylamino)thiazolidin4-one (13-20)

Methode A: A mixture of compounds (3-10) $(1.0 \mathrm{mmol})$ and thioglycolic acid $(1.0 \mathrm{mmol})$ in dry dioxane $(20 \mathrm{~mL})$ was refluxed for $24 \mathrm{~h}$. The volume was reduced to its half, and was left to cool. The formed precipitate was filtered off, dried and recrystallized from appropriate solvent to give target compounds. In case of no precipitate formed upon cooling, the solution was added to water and neutralized using $\mathrm{Na}_{2} \mathrm{CO}_{3}$ solution, the formed precipitate was filtered, dried and recrystallized to obtain the expected thiazolidinone products (13-20), respectively.

Method B: A mixture of 2-hydrazinylquinoline 2 (5 mmol, $0.796 \mathrm{~g}$ ), substituted aldehydes (5 $\mathrm{mmol}$ ) and thioglycolic acid (5 mmol; $0.460 \mathrm{~g}$ ) was taken in a round bottom flask containing 1,4-dioxane $(30 \mathrm{~mL})$ and equipped with a reflux condenser. The reaction mixture was refluxed for $24 \mathrm{~h}$ (monitored by TLC). The mixture was cooled to room temperature and the formed precipitate was filtered off and recrystalized from ethanol to give the expected thiazolidinone derivatives (13-20).

\section{2-(4-chlorophenyl)-3-(quinolin-2-ylamino) thiazolidin-4-one (13)}

Pale orange solid; yield $82.5 \%$; mp 178-180 ${ }^{\circ} \mathrm{C}$; IR (KBr, cm $\left.{ }^{-1}\right): 3433(\mathrm{NH}), 3050\left(\mathrm{CH}_{\text {arom }}\right)$, $2921(\mathrm{CH}$ streching $), 1705(\mathrm{C}=\mathrm{O}), 1607(\mathrm{C}=\mathrm{N})$; MS (m/z): $355\left[\mathrm{M}^{+}, 0.05 \%\right], 321\left[\mathrm{M}^{+}-\mathrm{Cl}, 0.12\right.$ $\%$ ], $245\left[\mathrm{M}^{+}-\mathrm{PhCl}, 0.17 \%\right], 213\left[\mathrm{~m}^{+}-\mathrm{C}_{9} \mathrm{H}_{8} \mathrm{~N}_{2}\right.$, $0.20 \%$ ], 144 [Base peak, $100 \%$ ]; ${ }^{1} \mathrm{H}$ NMR $\left(\mathrm{DMSO}_{\mathrm{d}}\right): \delta(\mathrm{ppm})=13.86(\mathrm{~s}, 1 \mathrm{H}, \mathrm{NH}), 8.93$ (s, $1 \mathrm{H}, \mathrm{CH}$, thiazolidinone), $8.2(\mathrm{~s}, 1 \mathrm{H}, 4-\mathrm{H}$, quinoline), 8.54, 8.52 and 7.86-7.47 (m, 9H, Ar$\mathrm{H}), 3.47$ (s, 2H, $\mathrm{CH}_{2}$, thiazolidinone). Anal. Calcd for $\mathrm{C}_{18} \mathrm{H}_{14} \mathrm{ClN}_{3} \mathrm{OS}(355.84)$ : $\mathrm{C}, 60.76 ; \mathrm{H}, 3.97 ; \mathrm{N}$, 11.81 Found: C, 60.61; H, 3.82; N, 11.66. 
2-(4-methoxyphenyl)-3-(quinolin-2-ylamino) thiazolidin-4-one (14)

Yellow solid; yield $74.5 \%$; mp $120-122{ }^{\circ} \mathrm{C}$; IR (KBr, cm $\left.{ }^{-1}\right)$ : ): $3430(\mathrm{NH}), 3049\left(\mathrm{CH}_{\text {arom }}\right), 2932$ $\left(\mathrm{CH}_{\text {streching }}\right), 2834\left(\mathrm{CH}_{3}\right) 1673(\mathrm{C}=\mathrm{O}), 1610(\mathrm{C}=\mathrm{N})$; MS (m/z): $351\left[\mathrm{M}^{+}, 1.7 \%\right], 337\left[\mathrm{M}^{+}-\mathrm{CH}_{3}, 2.82\right.$ \%], 245 [M+-Ph-O-CH, $2.54 \%$ ], 274 [Base peak, $100 \%$ ]; ${ }^{1} \mathrm{H}$ NMR $($ DMSO-d $): \delta(\mathrm{ppm})=13.78$ (s, 1H, NH), 8.91 (s, 1H, CH, thiazolidinone), 8.3 (s, 1H, 4-H, quinoline), 7.96-7.43 (m, 9H, Ar-H), $3.45\left(\mathrm{~s}, 2 \mathrm{H}, \mathrm{CH}_{2}\right.$, thiazolidinone). Anal. Calcd for $\mathrm{C}_{19} \mathrm{H}_{17} \mathrm{~N}_{3} \mathrm{O}_{2} \mathrm{~S}$ (351.42): $\mathrm{C}, 64.94 ; \mathrm{H}, 4.88 ; \mathrm{N}$, 11.96.Found: C, 64.79; H, 4.73; N, 11.81.

2-(4-(dimethylamino)phenyl)-3-(quinolin-2ylamino)thiazolidin-4-one (15)

Dark orange solid; yield 78.1\%; mp 180-182 ${ }^{\circ} \mathrm{C}$; IR ( $\left.\mathrm{KBr}, \mathrm{cm}^{-1}\right): 3429(\mathrm{NH}), 3050\left(\mathrm{CH}_{\text {arom }}\right)$, $2921\left(\mathrm{CH}_{\text {streching }}\right), 2855\left(2 \mathrm{CH}_{3}\right), 1649(\mathrm{C}=\mathrm{O}), 1605$ $(\mathrm{C}=\mathrm{N}) ; \mathrm{MS}(\mathrm{m} / \mathrm{z}): 364\left[\mathrm{M}^{+}, 0.13 \%\right], 365\left[\mathrm{M}^{+}+1\right.$, $0.06 \%$ ], 144 [Base peak, $100 \%$ ]. Anal. Calcd for $\mathrm{C}_{20} \mathrm{H}_{20} \mathrm{~N}_{4} \mathrm{OS}$ (364.47): C, 65.91; H, 5.53; N, 15.37. Found: C, 65.76; H, 5.38; N, 15.22.

\section{2-(2-chlorophenyl)-3-(quinolin-2-ylamino)} thiazolidin-4-one (16)

Pale yellow solid; yield 59.6 \%; mp 298-300 ${ }^{\circ} \mathrm{C}$; IR $\left(\mathrm{KBr}, \mathrm{cm}^{-1}\right): 3407(\mathrm{NH}), 3048\left(\mathrm{CH}_{\text {arom }}\right)$, $2909\left(\mathrm{CH}_{\text {streching }}\right), 1650(\mathrm{C}=\mathrm{O}), 1607(\mathrm{C}=\mathrm{N})$; MS $(\mathrm{m} / \mathrm{z}):$ : $355\left[\mathrm{M}^{+} 0.42 \%\right], 321\left[\mathrm{M}^{+}-\mathrm{Cl}, 0.13\right.$ \%], $245\left[\mathrm{M}^{+}-\mathrm{PhCl}, 2.31 \%\right], 213\left[\mathrm{~m}^{+}-\mathrm{C}_{9} \mathrm{H}_{8} \mathrm{~N}_{2}\right.$, $0.74 \%$ ], 144 [Base peak, $100 \%$ ]; ${ }^{1} \mathrm{H}$ NMR $\left(\mathrm{DMSO}_{\mathrm{6}}\right): \delta(\mathrm{ppm})=13.86(\mathrm{~s}, 1 \mathrm{H}, \mathrm{NH}), 8.93$ (s, 1H, CH, thiazolidinone), $8.2(\mathrm{~s}, 1 \mathrm{H}, 4-\mathrm{H}$, quinoline), 8.56, 8.53 and 7.87-7.45 ( $\mathrm{m}, 10 \mathrm{H}$, Ar$\mathrm{H}), 3.45$ (s, 2H, $\mathrm{CH}_{2}$, thiazolidinone). Anal. Calcd for $\mathrm{C}_{18} \mathrm{H}_{14} \mathrm{ClN}_{3} \mathrm{OS}(355.84)$ : C, 60.76; H, 3.97; N, 11.81. Found: C, 60.61; H, 3.82; N, 11.66.

2-phenyl-3-(quinolin-2-ylamino)thiazolidin-4one (17)

Yellow solid; yield $62.5 \%$; mp $140-142{ }^{\circ} \mathrm{C}$; IR (KBr, cm $\left.{ }^{-1}\right): 3438(\mathrm{NH}), 3050\left(\mathrm{CH}_{\text {arom }}\right), 2921$ $\left(\mathrm{CH}_{\text {streching }}\right), 1695(\mathrm{C}=\mathrm{O}), 1605(\mathrm{C}=\mathrm{N}) ; \mathrm{MS}(\mathrm{m} / \mathrm{z})$ : $321\left[\mathrm{M}^{+}, 0.07 \%\right], 320\left[\mathrm{M}^{+}-1,0.26 \%\right], 245\left[\mathrm{M}^{+}-\right.$ $\mathrm{Ph}, 1.53 \%$ ], 179 [ $\mathrm{M}^{+}-\mathrm{C}_{9} \mathrm{H}_{8} \mathrm{~N}_{2}, 1.53 \%$ ], 144 [Base peak, $100 \%$ ]; ${ }^{1} \mathrm{H}$ NMR $\left(\mathrm{DMSO}-\mathrm{d}_{6}\right): \delta(\mathrm{ppm})=$ 12.02 (s, 1H, NH), 8.2 (s, 1H, 4-H, quinoline), 8.11 (s, 1H, CH, thiazolidinone) 7.79-7.27 (m, $9 \mathrm{H}, \mathrm{Ar}-\mathrm{H}), 3.67$ ( $\mathrm{s}, 2 \mathrm{H}, \mathrm{CH}_{2}$, thiazolidinone). Anal. Calcd for $\mathrm{C}_{18} \mathrm{H}_{15} \mathrm{~N}_{3} \mathrm{OS}$ (321.40): C, 67.27; H, 4.70; N, 13.07. Found: C, 67.12; H, 4.55; N, 12.92 .

2-(2-hydroxyphenyl)-3-(quinolin-2-ylamino) thiazolidin-4-one (18) brown solid; yield $68.3 \%$; mp $263-265{ }^{\circ} \mathrm{C}$; IR $\left(\mathrm{KBr}, \mathrm{cm}^{-1}\right): 3652(\mathrm{OH}), 3427(\mathrm{NH}), 3072$ $\left(\mathrm{CH}_{\text {arom }}\right), 2979(\mathrm{CH}$ streching $), 1644(\mathrm{C}=\mathrm{O}), 1605$ $(\mathrm{C}=\mathrm{N}) ; \operatorname{MS}(\mathrm{m} / \mathrm{z}): 336\left[\mathrm{M}^{+}-1,0.06 \%\right], 320$ $\left[\mathrm{M}^{+}-\mathrm{OH}, 0.10 \%\right], 245\left[\mathrm{M}^{+}-\mathrm{PhOH}, 2.95 \%\right]$, 144 [Base peak, $100 \%$ ]; ${ }^{1} \mathrm{H}$ NMR (DMSO-d $)$ : $\delta(\mathrm{ppm})=13.86(\mathrm{~s}, 1 \mathrm{H}, \mathrm{NH}), 11.4(\mathrm{~s}, 1 \mathrm{H}, \mathrm{OH})$, 8.93 (s, 1H, CH, thiazolidinone), $8.2(\mathrm{~s}, 1 \mathrm{H}, 4-\mathrm{H}$, quinoline), 8.56, 8.53 and 7.87-7.45 (m, 9H, Ar$\mathrm{H}), 3.45$ (s, 2H, $\mathrm{CH}_{2}$, thiazolidinone). Anal. Calcd for $\mathrm{C}_{18} \mathrm{H}_{15} \mathrm{~N}_{3} \mathrm{O}_{2} \mathrm{~S}$ (337.40): $\mathrm{C}, 64.08 ; \mathrm{H}, 4.48 ; \mathrm{N}$, 12.45. Found: C, 63.92; H, 4.33; N, 12.32 .

\section{2-(3-nitrophenyl)-3-(quinolin-2-ylamino) thiazolidin-4-one (19)}

Yellow solid; yield $74.05 \%$; mp $255-257{ }^{\circ} \mathrm{C}$; IR $\left(\mathrm{KBr}, \mathrm{cm}^{-1}\right): 3431(\mathrm{NH}), 3012\left(\mathrm{CH}_{\text {arom }}\right), 2902$ $\left(\mathrm{CH}_{\text {streching }}\right), 1722(\mathrm{C}=\mathrm{O}), 1647(\mathrm{C}=\mathrm{N}), 1514,1342$ $\left(\mathrm{NO}_{2}\right) ; \mathrm{MS}(\mathrm{m} / \mathrm{z}): 366\left[\mathrm{M}^{+}, 0.05 \%\right], 322\left[\mathrm{M}^{+}-\right.$ $\mathrm{NO}_{2}, 0.03 \%$, ], $355\left[\mathrm{M}^{+}-\mathrm{PhNO}_{2}, 0.08 \%\right.$ ],76 [Base peak, $100 \%$ ]; ${ }^{1} \mathrm{H}$ NMR (DMSO-d 6 ): $\delta(\mathrm{ppm})=$ 12.02 (s, 1H, NH), 8.3 (s, 1H, 4-H, quinoline), 8.2 (s, 1H, CH, thiazolidinone) 8.1-7.22 (m, 9H, Ar$\mathrm{H}), 3.7$ (s, 2H, $\mathrm{CH}_{2}$, thiazolidinone). Anal. Calcd for $\mathrm{C}_{18} \mathrm{H}_{14} \mathrm{~N}_{4} \mathrm{O}_{3} \mathrm{~S}$ (366.40): 59.01; $\mathrm{H}, 3.85 ; \mathrm{N}$, 15.29. Found: 58.87 ; H, 3.725; N, 15.13 .

\section{2-(naphthalen-1-yl)-3-(quinolin-2-ylamino) thiazolidin-4-one (20)}

Pale yellow solid; yield $72.6 \%$; mp 260-262 ${ }^{\circ} \mathrm{C}$; IR $\left(\mathrm{KBr}, \mathrm{cm}^{-1}\right): 3426(\mathrm{NH}), 3048\left(\mathrm{CH}_{\text {arom }}\right)$, $2917\left(\mathrm{CH}_{\text {streching }}\right), 1648(\mathrm{C}=\mathrm{O}), 1605(\mathrm{C}=\mathrm{N})$; MS $(\mathrm{m} / \mathrm{z}): 369\left[\mathrm{M}^{+}-2,0.05 \%\right.$ ], 144 [Base peak, $100 \%$ ]. Anal. Calcd for $\mathrm{C}_{22} \mathrm{H}_{17} \mathrm{~N}_{3} \mathrm{OS}$ (371.46): C, 71.14; H, 4.61; N, 11.31. Found: C, 71.01; H, $4.48 ; \mathrm{N}, 11.18$.

General procedures for the synthesis of 1-substituted 3'-(quinolin-2-ylamino) spiro[indoline-3,2'-thiazolidine]-2,4'-diones (21, 22)

Method A: a mixture of compound 11 or 12 $(1.0 \mathrm{mmol})$ and thioglycolic acid $(1.0 \mathrm{mmol})$ in dry dioxane $(20 \mathrm{~mL})$ was refluxed for $24 \mathrm{~h}$. The volume was reduced to its half, and was left to cool. The formed precipitate was filtered off, dried and recrystallized from appropriate solvent to give target compounds $\mathbf{2 1}$ and $\mathbf{2 2}$.

Method B: A mixture of 2-hydrazinylquinoline 2 (5 mmol, $0.796 \mathrm{~g})$, isatin or methyl isatin (5 $\mathrm{mmol}$ ) and thioglycolic acid (5 mmol; $0.460 \mathrm{~g}$ ) was taken in a round bottom flask containing 1,4-dioxane $(30 \mathrm{~mL})$ and equipped with a reflux condenser. The reaction mixture was refluxed for $24 \mathrm{~h}$ (monitored by TLC). The mixture was cooled 
to room temperature and the formed precipitate was filtered off and recrystallized from ethanol to give 1-substituted 3'-(quinolin-2-ylamino) spiro[indoline-3,2'-thiazolidine]-2,4'-dione 21 and 22.

3'-(quinolin-2-ylamino)spiro[indoline-3,2'thiazolidine]-2,4'-dione (21)

Brown solid; yield $69.8 \%$; mp 298-300 ${ }^{\circ} \mathrm{C}$; IR (KBr, cm $\left.{ }^{-1}\right): 3439$ ( $\left.\mathrm{NH}_{\text {hydrazone }}\right), 3217$ $\left(\mathrm{NH}_{\text {indole }}\right), 3059\left(\mathrm{CH}_{\text {arom }}\right), 2923\left(\mathrm{CH}_{\text {streching }}\right), 1701$ $\left(\mathrm{C}=\mathrm{O}_{\text {thiazole }}\right), 1645\left(\mathrm{C}=\mathrm{O}_{\text {indole }}\right), 1605(\mathrm{C}=\mathrm{N}) ; \mathrm{MS}$ $(\mathrm{m} / \mathrm{z}): 361\left[\mathrm{M}^{+}-1,0.08 \%\right.$ ] $363\left[\mathrm{M}^{+}+1,0.23 \%\right.$ ], 259 [Base peak, $100 \%$ ]; ${ }^{1} \mathrm{H}$ NMR (DMSO-d ${ }_{6}$ ): $\delta(\mathrm{ppm})=13.01(\mathrm{~s}, 1 \mathrm{H}, \mathrm{NH}), 12.4(\mathrm{~s}, 1 \mathrm{H}, \mathrm{NH}$ indole), 8.4 (s, 1H, 4-H, quinoline), 7.9-7.1 (m, $9 \mathrm{H}, \mathrm{Ar}-\mathrm{H}), 3.64\left(\mathrm{~m}, 2 \mathrm{H}, \mathrm{CH}_{2}\right.$, thiazolidinone). Anal. Calcd for $\mathrm{C}_{19} \mathrm{H}_{14} \mathrm{~N}_{4} \mathrm{O}_{2} \mathrm{~S}$ (362.41): C, 62.97; H, 3.89; N, 15.46. Found: C, 62.82; H, 3.74; N, 15.31 .

1 - methyl-3'-(quinolin-2-ylamino) spiro[indoline-3,2'-thiazolidine]-2,4'-dione (22)

Orange solid; yield $72.2 \%$; mp 196-198 ${ }^{\circ} \mathrm{C}$; IR (KBr, cm $\left.{ }^{-1}\right): 3432\left(\mathrm{NH}_{\text {hydrazone }}\right), 3050$ $\left(\mathrm{CH}_{\text {arom }}\right), 2923(\mathrm{CH}$ streching $), 1728\left(\mathrm{C}=\mathrm{O}_{\text {thiazole }}\right)$, $1677\left(\mathrm{C}=\mathrm{O}_{\text {indole }}\right), 1604(\mathrm{C}=\mathrm{N}) ; \mathrm{MS}(\mathrm{m} / \mathrm{z}): 376$ $\left[\mathrm{M}^{+}, 0.09 \%\right.$ ], 273 [Base peak, $100 \%$ ]; ${ }^{1} \mathrm{H}$ NMR $\left(\mathrm{DMSO}_{\mathrm{d}}\right): \delta(\mathrm{ppm})=13.01(\mathrm{~s}, 1 \mathrm{H}, \mathrm{NH}), 8.4(\mathrm{~s}$, 1H, 4-H, quinoline), 7.94-7.07 (m, 9H, Ar-H), $3.66\left(\mathrm{~m}, 2 \mathrm{H}, \mathrm{CH}_{2}\right.$, thiazolidinone), $3.45(\mathrm{~s}, 3 \mathrm{H}$, $\mathrm{CH}_{3}$ ). Anal. Calcd for $\mathrm{C}_{20} \mathrm{H}_{16} \mathrm{~N}_{4} \mathrm{O}_{2} \mathrm{~S}$ (376.43): C, 63.81; H, 4.28; N, 14.88. Found: C, 63.66; H, 4.13; N, 14.73.

\section{Pharmacology \\ Anti-inflammatory activity}

Male Wistar rats weighing (120-150 g) were used throughout the assay. Animals were housed under standardized conditions of light and temperature and received standard rat chow and tap water ad libitum. Animals were randomly assigned to different experimental groups, each of six rats and kept in separate cages. One group of six rats was kept as a control group and another group received the standard drug indomethacin. All animal procedures were performed after an approval from the Ethics Committee of the National Research Centre and in accordance with the recommendations for the proper care and use of laboratory animals (NIH publication No. 85-23, revised 1985). Carrageenan lambda from Sigma Aldrich Chemical Co. (USA), indomethacin from Khahira Pharmaceutical, and Chemical Co. (Cairo, Egypt). Paw edema was induced by subplantar injection of $100 \mu \mathrm{L}$ of 1 $\%$ sterile carrageenan in saline into the right hind paw $(1 \%$ suspension of carrageenan in sterile saline was prepared, the suspension was placed in a refrigerator $\left(4{ }^{\circ} \mathrm{C}\right)$ overnight to allow complete hydration of the carrageenan [32]. Twelve groups of rats, each of six animals, were used. One group received saline and served as control. Indomethacin $(10 \mathrm{mg} / \mathrm{kg})$ was administered to a group of rats that served as a positive control. Tested groups received the compounds in a dose of $(10 \mathrm{mg} / \mathrm{kg})$. All the tested compounds and indomethacin were orally administered $1 \mathrm{~h}$ before induction of inflammation. The right hind paw volume was measured immediately before carrageenan injection and at selected times $(1,2$, 3 , and $4 \mathrm{~h}$ ) thereafter by planimeter [33].

\section{Analgesic activity}

Each animal was placed gently on a hot plate at $50^{\circ} \mathrm{C}$. Latency to exhibit nociceptive responses, such as licking paws or jumping off the hot plate was determined 30, 60, 90 min after administration of test substances or saline [34]. All drugs were injected orally $(100 \mathrm{mg} / \mathrm{kg}) 30$ minutes before placing the animal on the hot plate. Aspirin (100 $\mathrm{mg} / \mathrm{kg}$ ) was administered to a group of rats that served as a positive control.

\section{$\underline{\text { Results and Discussion }}$}

Synthesis of target compounds (13-22) was achieved as outlined in Schemes 1 and 2. The starting material 2-hydrazinylquinoline $\mathbf{2}$ was synthesized in a high yield from the reaction of hydrazine hydrate with 2-chloroquinoline 1 in refluxing n-butanol, through procedures previously reported [35]. Reaction of compound 2 with substituted aldehydes in (1:1) molar ratio with elimination of water afforded the corresponding arylidenehydrazinyl quinoline derivatives 3-10 in a good yield. Structures of compounds $\mathbf{3 - 1 0}$ were supported by their elemental analysis and spectral data. ${ }^{1}$ HNMR spectra of compounds $\mathbf{3}-\mathbf{1 0}$ showed the presence of the $(\mathrm{NH})$ proton and azomethine $(\mathrm{CH}=\mathrm{N})$ proton signals at the expected regions. ${ }^{1}$ HNMR spectra of compounds 3-10 Showed singlet signals at the ranges $\delta$ 13.3-11.2 ppm corresponding to $\mathrm{NH}$ protons and singlet signals at the ranges $\delta 9.0-8.49 \mathrm{ppm}$ corresponding to the azomthine protons. Furthermore, derivatives $\mathbf{4 ,} \mathbf{5}$, and $\mathbf{1 2}$ showed singlet signals at the ranges $\delta$ 3.02-3.8 ppm due to methyl group protons. Derivative 8 showed a singlet signal at $11.2 \mathrm{ppm}$ corresponding to the hydroxyl group proton. 
IR spectra of compounds 3-12 revealed the presence of absorption bands at $3431-3393 \mathrm{~cm}^{-1}$ due to NH, 3106-3043 $\mathrm{cm}^{-1}$ corresponding to $\mathrm{CH}$ Aromatic and $1663-1599 \mathrm{~cm}^{-1}$ corresponding to $\mathrm{C}=\mathrm{N}$. Furthermore, IR spectrum of compound 4 and $\mathbf{5}$ showed the presence of $\mathrm{CH}$ aliphatic at 2966 and $2911 \mathrm{~cm}^{-1}$ respectively. Also, IR spectrum of compound $\mathbf{8}$ showed the presence of $\mathrm{OH}$ group at $3752 \mathrm{~cm}^{-1}$. On the other hand IR spectrum of compound 9 showed presence of $\mathrm{NO}_{2}$ at 1518 and $1347 \mathrm{~cm}^{-1}$. IR spectra of compounds $\mathbf{1 1}$ and $\mathbf{1 2}$ showed additional absorption bands at 1646 and $1674 \mathrm{~cm}^{-1}$ respectively, due to $\mathrm{C}=\mathrm{O}$ groups. Mass spectra of all compounds showed the molecular ion peaks which were in agreement with their molecular formulae.

Condensation of derivatives 3-12 with thioglycolic acid in dry dioxane led to the formation of the corresponding thiazolidinone derivatives 13-22. IR spectra of the thiazolidinone compounds 13-20, revealed the appearance of new absorption bands at 1722-1644 $\mathrm{cm}^{-1}$ attributed to $\mathrm{C}=\mathrm{O}$ functionalities, while their ${ }^{1} \mathrm{H}$ NMR spectra showed singlet signals at the ranges of $\delta 3.7-3.45 \mathrm{ppm}$ and $\delta 8.93-8.11$, representing the corresponding methylene protons $\left(-\mathrm{CH}_{2}^{-}\right)$and the methine protons (N-CH-S) of the new formed thiazolidinone ring. On the other hand, IR spectra of derivatives $\mathbf{2 1}$ and $\mathbf{2 2}$ showed absorption bands at 1701 and $1028 \mathrm{~cm}^{-1}$ due to presence of carbonyl groups, and their ${ }^{1} \mathrm{HNMR}$ spectra showed multiplet signals at $\delta 3.64 \mathrm{ppm}$ and $\delta 3.66$ representing the corresponding methylene protons $\left(-\mathrm{CH}_{2}-\right)$ and there were no signals due to the methine protons (N-CH-S) of the new formed spiro[indoline-3,2'thiazolidine]-2,4'-dione derivatives. Mass spectra of the compounds showed the molecular ion peaks which were in agreement with their molecular formula. One-pot three component reaction was adopted for synthesis of the target compound 13-22 involving 2-hydrazinylquinoline, aromatic aldehyde or isatin derivatives and thioglycolic acid. The reaction has been suggested to proceed via imine formation followed by the attack of the sulfur nucleophile on the imine carbon, followed by intramolecular cyclization with the elimination of water to give thiazolidin-4-one derivatives as discussed in Scheme 3 [36]. The one-pot reaction gives a satisfactory yield compared to the multistep reaction and have the merit of saving more time in the synthesis process.
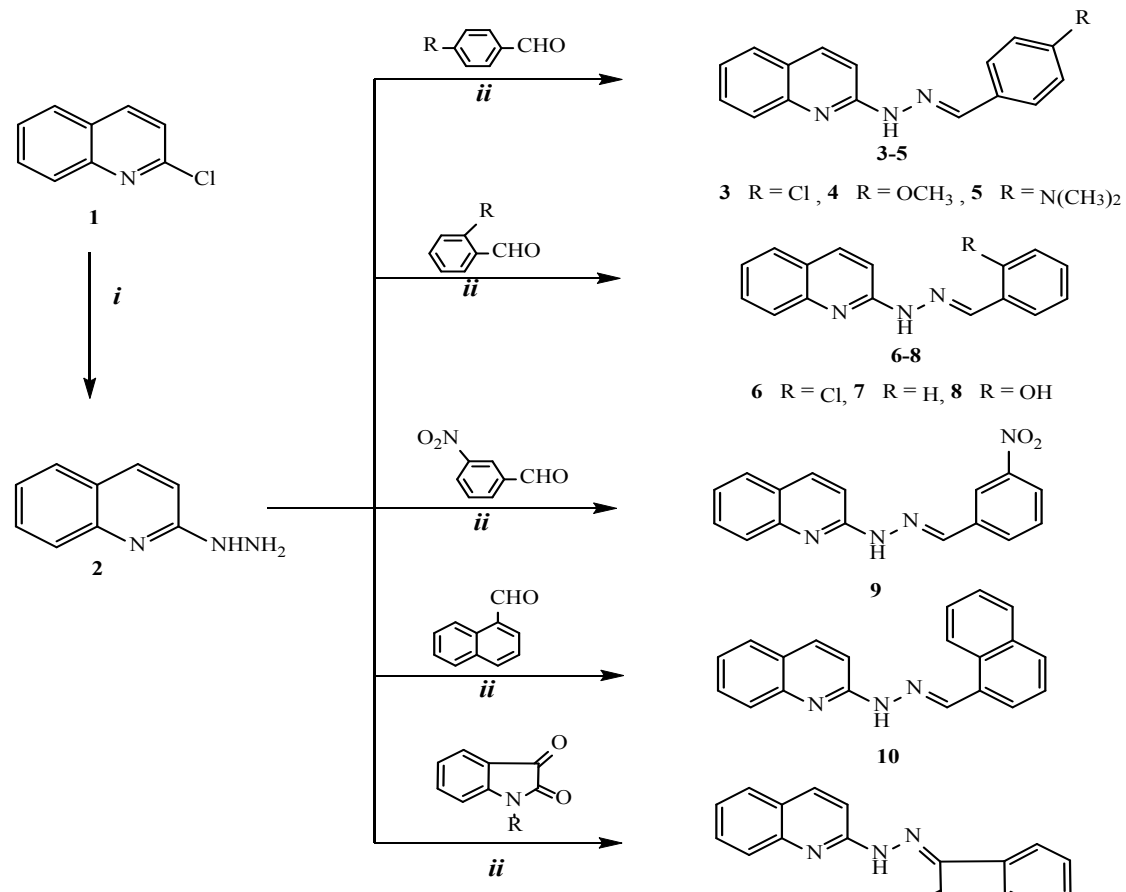<smiles>C(=N/Nc1ccc2ccccc2n1)\c1cccc2ccccc12</smiles>

10

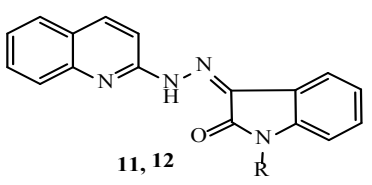

$11 \mathrm{R}=\mathrm{H}, 12 \mathrm{R}=\mathrm{CH}_{3}$

Scheme 1 Synthesis of derivatives 2-12.

Reagents and conditions $i \mathrm{BuOH} / \mathrm{NH}_{2} \mathrm{NH}_{2} \mathrm{H}_{2} \mathrm{O} /$ Reflux, 6h, $\boldsymbol{i}$ EtOH/Acetic acid/reflux $3 \mathrm{~h}$

Egypt.J.Chem. Special Issue (2018) 

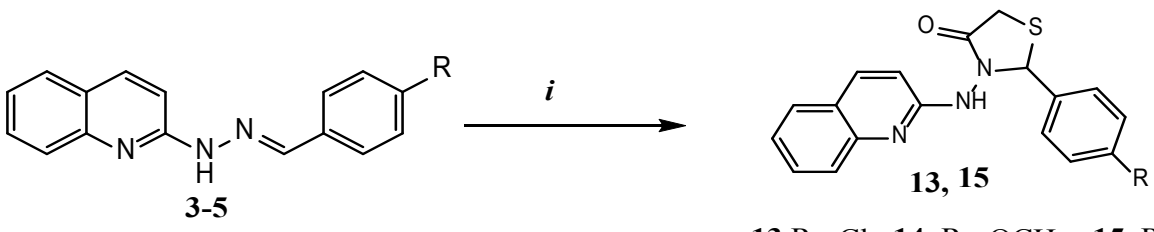

$3 \mathrm{R}=\mathrm{Cl}, 4 \mathrm{R}=\mathrm{OCH}_{3}, 5 \mathrm{R}=\mathrm{N}\left(\mathrm{CH}_{3}\right)_{2}$

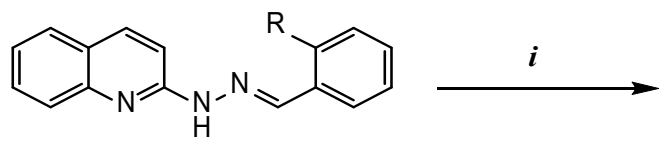

$13 \mathrm{R}=\mathrm{Cl}, 14 \mathrm{R}=\mathrm{OCH}_{3}, 15 \mathrm{R}=\mathrm{N}\left(\mathrm{CH}_{3}\right)_{2}$

$$
\text { 6-8 }
$$

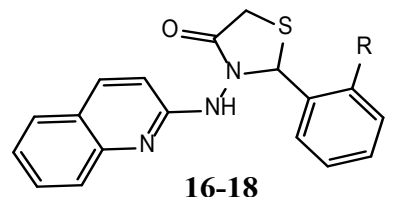

$16 \mathrm{R}=\mathrm{Cl}, 17 \mathrm{R}=\mathrm{H}, \mathbf{1 8} \mathrm{R}=\mathrm{OH}$

$6 \mathrm{R}=\mathrm{Cl}, 7 \mathrm{R}=\mathrm{H}, 8 \mathrm{R}=\mathrm{OH}$

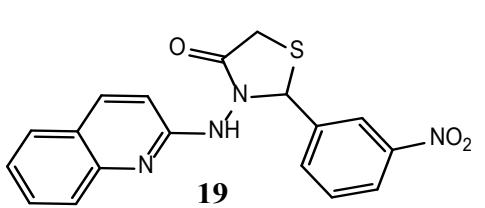

9
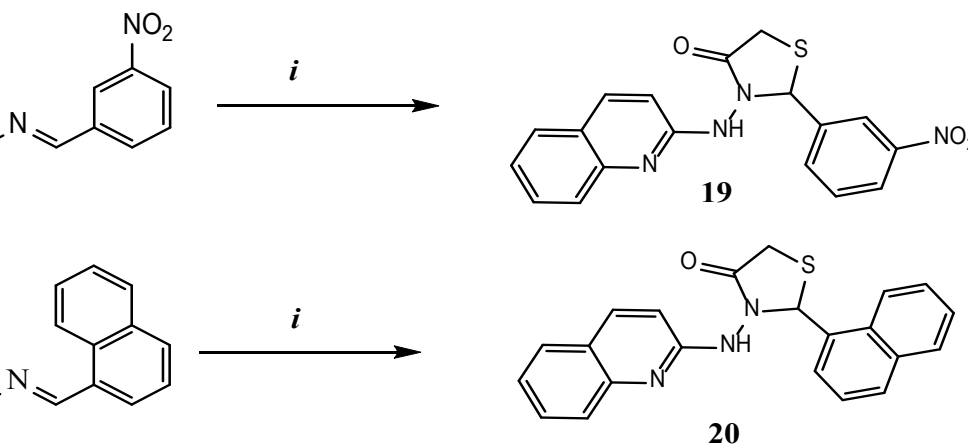

10

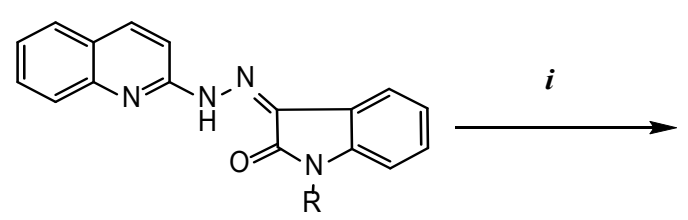

11, 12

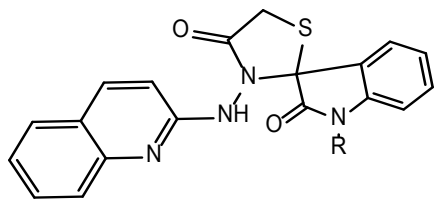

21,22

$11 \mathrm{R}=\mathrm{H}, 12 \mathrm{R}=\mathrm{CH}_{3}$

$21 \mathrm{R}=\mathrm{H}, 22 \mathrm{R}=\mathrm{CH}_{3}$

Scheme 2 Synthesis of derivatives 13-22.

Reagents and conditions $i \mathrm{SHCH}_{2} \mathrm{COOH} / 1,4-$ Dioxane/Reflux, 24h

$$
\text { + }
$$

Scheme 3 formation of compounds 13 - 22 


\section{Anti-inflammatory activity}

In this study, 10 newly synthesized derivatives were evaluated for their anti-inflammatory activity by using carrageenan-induced paw edema bioassay in rats [32] using indomethacin as a reference standard. Results were expressed as mean \pm SE. The difference between control and treated groups were tested using Two way Anova followed by LST test. The anti-inflammatory results (Table 1) revealed that the highest antiinflammatory potency at $4 \mathrm{~h}$ was gained by 6 derivatives according to the following order $22>$ $\mathbf{1 7}>\mathbf{1 3}>\mathbf{1 4}>\mathbf{2 1}>\mathbf{1 5}$. It has been noticed that they exhibited early action showing good percentages of edema inhibition relative to the reference drug indomethacin. Compound 22 carrying indole ring system inhibited the edema volume significantly at the ${ }^{1} \mathrm{st} h$ post administration, and the activity was enhanced up to the 4 th $\mathrm{h}$ giving promising edema volume inhibition compared to that produced by indomethacin. Also, significant edema inhibition at the first hour post compounds administration was observed by derivatives $22,17,21,16$ and 15, respectively. Regarding Structure activity relationship (SAR), it is clear that presence of $\mathrm{Cl}$ at position 4 of the phenyl ring of compound 13 has a significant effect on edema inhibition more than that of $\left(-\mathrm{OCH}_{3}\right)$ and $-\mathrm{N}\left(\mathrm{CH}_{3}\right)_{2}$ in compounds 14 and 15 respectively. On the other hand, absence of substitution on the phenyl ring of compound $\mathbf{1 7}$ leads to higher level of edema inhibition more than compounds $\mathbf{1 6}$ and 18. It can be noticed that presence of $\left(\mathrm{NO}_{2}\right)$ group at position 2 of the phenyl ring of compound $\mathbf{1 9}$ has no significant effect on level of edema inhibition. A good inhibition level relative to the reference drug indomethacin was observed by the N-methyl indole derivative 22 .

TABLE 1. Anti-inflammatory effect of the tested compounds on carrageenan-induced rat paw edema $(\mathrm{mL})$

\begin{tabular}{|c|c|c|c|c|}
\hline \multirow[t]{2}{*}{ Group no. } & \multicolumn{4}{|c|}{ Paw odema volume (mL) } \\
\hline & lh & $2 \mathrm{~h}$ & $3 \mathrm{~h}$ & 4h \\
\hline Control & $1429 \pm 13.31$ & $99.09+3.23$ & $79.83+1.94$ & $60.07 \pm 6.98$ \\
\hline Indomethacin $10 \mathrm{mg} / \mathrm{kg}$ & $42.69 \pm 6.66^{*}$ & $38.02+4.01^{*}$ & $34.70+3.94^{*}$ & $26.06+4.11^{*}$ \\
\hline 13 & $7481 \pm 7.75^{\circ}$ & $6216 \pm 6.04^{*}$ & $57.61 \pm 296^{*}$ & $33,45 \pm 7.32$ \\
\hline 14 & $79.10+523^{\circ}$ & $69.63+293^{*}$ & $31.36+2.68^{*}$ & $31.82+2.23^{\circ}$ \\
\hline 15 & $8267 \pm 11.11^{*}$ & $64.88+6.28^{+}$ & $39.23+3.03^{*}$ & $29.58 \pm 3.44^{\circ}$ \\
\hline 16 & $103,3 \pm 608$ & $71,44+3,16$ & $5290+4.43^{*}$ & $28.59 \pm 2.23^{\circ}$ \\
\hline 17 & $5995+257^{*}$ & $5242+262^{*}$ & $39.24+4.03^{*}$ & $24.29 \pm 3.86^{\circ}$ \\
\hline 18 & $108.4 \pm 6.72$ & $88.16+5.03$ & $63.50+4.12$ & $33.04+2.94^{\circ}$ \\
\hline 19 & $119.8 \pm 8.49$ & $90.63 \pm 6.22$ & $3299+2.8 ?$ & $36.66+2.98$ \\
\hline 20 & $1123 \pm 989$ & $86.64 \pm 8.26$ & $68.66 \pm 2.08$ & $389 \pm 3.14$ \\
\hline 21 & $7960 \pm 566^{\circ}$ & $60.49 \pm 5.99^{\circ}$ & $47.72+523^{*}$ & $26.50 \pm 3.14^{*}$ \\
\hline 22 & $4285 \pm 5.94^{\circ}$ & $36.26 \pm 4.11^{*}$ & $30.54 \pm 1.85^{\circ}$ & $18.00 \pm 2.01^{\circ}$ \\
\hline
\end{tabular}

The data represent the mean \pm standard error of the mean $(n=6)$.

Values represent the mean \pm S.E. of six animals for each group.

$* \mathrm{P}<0.05$ : Statistically significant from Control. (Two way Anova followed by LST test).

\begin{abstract}
Analgesic activity
The analgesic activity of the above mentioned derivatives was also evaluated in comparison with aspirin as a standard reference drug $(100 \mathrm{mg} / \mathrm{kg})$ by applying hot plate test [34]. The results were expressed as mean $\pm \mathrm{SE}$. The difference between the control and treatment groups was tested using two way ANOVA followed by LST test. The analgesic activity expressed in Table 2 showed that the longest duration of action up to 90 min post compounds administration was obtained by the compounds 13, 17 and 22. They exhibited good analgesia relative to that obtained by aspirin. Unsubstituted phenyl ring in case of compound $\mathbf{1 7}$ has a noticeable effect on analgesic activity more than that having $-\mathrm{Cl}$ at position 4 in compound 13. It is clear that the presence of $\mathrm{N}$-methyl group on the indole ring give higher activity, this in turn reveals that there is a
\end{abstract}

Egypt.J.Chem. Special Issue (2018) significant relation between activity and substituent on the nitrogen atom of indole nucleus.

\section{Conclusion}

In summary, we have discussed the synthesis of some quinoline based thiazolidinone derivatives in a three-step process. All newly synthesized compounds have been fully characterized. Furthermore, all new thiazolidinone derivatives were evaluated for their antiinflammatory and analgesic activity. The Study results revealed that the highest anti-inflammatory potency was gained by $\mathbf{6}$ derivatives, showing a good edema inhibition compared to the reference drug indomethacin. The longest duration of analgesic action up to $90 \mathrm{~min}$ post compounds administration was obtained by three compounds, they exhibited potent analgesia compared to that obtained by aspirin. 
TABLE 2. Analgesic activity of the tested compounds by hot plate method.

\begin{tabular}{|c|c|c|c|c|}
\hline \multirow[t]{2}{*}{ Group no. } & \multicolumn{4}{|c|}{ Reaction time (sec.) } \\
\hline & $0 \min$ & $30 \min$ & $60 \mathrm{~min}$ & $90 \mathrm{~min}$ \\
\hline Control & $5.122+0.37$ & $5.520+0.22$ & 5.24040 .45 & $5.040+0.41$ \\
\hline Aspirin $100 \mathrm{mg} / \mathrm{kg}$ & $5.100+0.58$ & $11.56+0.69^{\mathrm{m}}$ & $14.98+1-41$ & $13.64+1.36$ \\
\hline 13 & $5.000 \pm 0.36$ & $6.525+0.68$ & $10.44 \pm 080^{\circ}$ & $12.33 \pm 0.85^{\circ}$ \\
\hline 14 & $4.100 \pm 029$ & 5.32040 .14 & $3,88040.54$ & 9.2800197 \\
\hline 15 & $5,075 \pm 0.4$ & 600040.70 & $6,825 \pm 0.55$ & $7.850 \pm 1.02$ \\
\hline 16 & $4.875+0.16$ & $5.425+0.52$ & $6.253 \pm 0.71$ & $6.575 \pm 0.96$ \\
\hline 17 & $4.550 \div 0.44$ & $8.240+0.94$ & $10.47 \pm 097^{*}$ & $10.38+1.13^{*}$ \\
\hline 18 & $4.775 \pm 0.35$ & $5.475 \pm 0.44$ & 5.75040 .63 & 600040.76 \\
\hline 19 & $4.060+037$ & $4.775+0.51$ & $6.775 \pm 0.6$ & $7.700+0.58$ \\
\hline 20 & $3.750+0.49$ & $5.350+0.26$ & $5.850+0.68$ & $6675 \pm 0.53$ \\
\hline 21 & $4.625+0.46$ & $5.775+0.51$ & $7.100+0.62$ & $8.275 \pm 0.57$ \\
\hline 22 & $5.525+0.6$ & $10.55+0.76^{*}$ & $10.10+1.10^{*}$ & $10.50+0.86^{\circ}$ \\
\hline
\end{tabular}

The data represent the mean \pm standard error of the mean $(n=6)$.

Values represent the mean \pm S.E. of six animals for each group.

$* \mathrm{P}<0.05$ : Statistically significant from control. (Two way Anova followed by LST test).

\section{References}

1. Hamama, W.S., Ibrahim, M.E., Gooda, A.A., Zoorob, H.H., Recent advances in the chemistry of 2-chloroquinoline-3-carbaldehyde and related analogs. RSC $A d v$., 8, 8484-8515 (2018).

2. Keri, R.S., Patil, S.A., Quinoline: A promising antitubercular target. Biomed. Pharmacother., 68, 1161-1175, (2014).

3. Nayyar, A., Jain, R., Synthesis and anti-tuberculosis activity of 2,4-disubstituted quinolines. Indian $J$. Chem., 47B, 117-128 (2008).

4. Vandekerckhove, S., Van Herreweghe, S., Willems, J., Danneels, B., Desmet, T., de Kock, C., Smith, P.J., Chibale, K., D'hooghe, M., Synthesis of functionalized 3-, 5-, 6- and 8 -aminoquinolines via intermediate (3-pyrrolin1-yl)- and (2-oxopyrrolidin-1-yl)quinolines and evaluation of their antiplasmodial and antifungal activity. Eur. J. Med. Chem., 92, 91-102 (2015).

5. Desai, N.C., Kotadiya, G.M., Trivedi, A.R., Studies on molecular properties prediction, antitubercular and antimicrobial activities of novel quinoline based pyrimidine motifs. Bioorg. Med. Chem. Lett., 24, 3126-3130 (2014).

6. Srivastava, A., Singh, M.K., Singh, R.M., Pyrazolo-fused quinoline analogues: Synthesis of 1H-pyrazolo [3, 4-b] quinolines and 3-amino$1 \mathrm{H}$-pyrazolo [3, 4-b] quinolines from 3-formyl and 3-cyano-2- chloroquinolines. Indian J. Chem. 45B, 292-296 (2006).

7. Spanò, V., Parrino, B., Carbone, A., Montalbano, A., Salvador, A., Brun, P., Vedaldi, D., Diana, P., Cirrincione, G., Barraja, P., Pyrazolo[3,4-h] quinolines promising photosensitizing agents in the treatment of cancer. Eur. J. Med. Chem., 102 334-351 (2015).

8. Pun, I.H.Y., Chan, D., Chan, S.H., Chung, P.Y., Zhou, Y.Y., Law, S., Lam, A.K.Y., Chui, C.H., Chan, A.S.C., Lam, K.H., Tang, J.C.O., Anticancer Effects of a Novel Quinoline Derivative 83b1 on Human Esophageal Squamous Cell Carcinoma through Down-Regulation of COX-2 mRNA and PGE2. Cancer Res. Treat., 49, 219229 (2017)

9. Pertz, H.H., Milhahn, H.C., Eich, E., Cycloalkanecarboxylic Esters Derived from Lysergol, Dihydrolysergol-I, and Elymoclavine as Partial Agonists and Antagonists at Rat 5-HT 2A Receptors: Pharmacological Evidence that the Indolo[4,3- fg ]quinoline System of the Ergolines Is Responsible for Hi. J. Med. Chem., 42, 659-668 (1999).

10. Sah, P. Garg, S. P. Nautiyal, S.R., Some new biologically active quinoline analogues. Indian $J$. Heterocycl. Chem., 7, 201-204 (1998).

11. Vandekerckhove, S., D'hooghe, M., Quinolinebased antimalarial hybrid compounds. Bioorg. Med. Chem., 23, 5098-5119 (2015).

12. Ahmed, N., Brahmbhatt, K.G., Sabde, S., Mitra, D., Singh, I.P., Bhutani, K.K., Synthesis and antiHIV activity of alkylated quinoline 2,4-diols. Bioorg. Med. Chem., 18, 2872-2879 (2010).

13. Karekal, M.R., Biradar, V., Bennikallu Hire Mathada, M., Synthesis, Characterization, Antimicrobial, DNA Cleavage, and Antioxidant Studies of Some Metal Complexes Derived from Schiff Base Containing Indole and Quinoline Moieties. Bioinorg. Chem. Appl., 2013, 315972, (2013). 
14. Wardakhan W., New Approaches for the Uses of 2-Cyanomethyl Thiazole in the Synthesis of Fused Heterocyclic Derivatives with Anti-Tumor Activities. Egypt. J. Chem., 54, 289-297 (2011).

15. Abd-Elzaher, M.M., Moustafa, S.A., Mousa, H.A., Labib, A.A., Ali, M.M., Synthesis, Characterization and Anticancer Activity of $\mathrm{La}(\mathrm{III}), \mathrm{Ce}(\mathrm{III}), \operatorname{Pr}(\mathrm{III})$ and $\mathrm{Gd}(\mathrm{III})$ Complexes of 1,1 - Bis(Z)-N- ethyldiene-5- Methylthiazol2-Amine Ferrocene. Egypt. J. Chem., 57, 59-73 (2014).

16. Ez El-Arab, E., El-Said, A.I., Amine, M.S., Moharram, H.H., Synthesis and Antitumor Activity Evaluation of New 2-(4-aminophenyl) benzothiazole/oxazole/imidazole Derivatives. Egypt. J. Chem., 59, 967-984 (2016).

17. Abdelazeem, A.H., Salama, S.A., Maghrabi, I.A., Design, Synthesis, and Anti-Inflammatory Evaluation of Novel DiphenylthiazoleThiazolidinone Hybrids. Arch. Pharm. (Weinheim)., 348, 518-530 (2015).

18. Hu, J., Wang, Y., Wei, X., Wu, X., Chen, G., Cao, G., Shen, X., Zhang, X., Tang, Q., Liang, G., Li, X., Synthesis and biological evaluation of novel thiazolidinone derivatives as potential anti-inflammatory agents. Eur. J. Med. Chem., 64, 292-301 (2013).

19. Deep, A., Narasimhan, B., Lim, S.M., Ramasamy, K., Mishra, R.K., Mani, V., 4-Thiazolidinone derivatives: synthesis, antimicrobial, anticancer evaluation and QSAR studies. RSC $A d v$., 6, 109485-109494 (2016).

20. Subhedar, D.D., Shaikh, M.H., Shingate, B.B., Nawale, L., Sarkar, D., Khedkar, V.M., Novel tetrazoloquinoline-thiazolidinone conjugates as possible antitubercular agents: synthesis and molecular docking. Medchemcomm, 7, 1832-1848 (2016).

21. Drăgan, M.W., Stan, C.D., Pânzariu, A., Jităreanu, A., Profire, L., New thiazolidine-4-ones of ferulic acid with antioxidant potential. Farmacia, 63, 4-8 (2015)

22. O'Neill, L.A.J., Targeting signal transduction as a strategy to treat inflammatory diseases. Nat. Rev. Drug Discov., 5, 549-563 (2006).

23. Cheeseright, T.J., Holm, M., Lehmann, F., Luik, S., Göttert, M., Melville, J.L., Laufer, S., Novel Lead Structures for p38 MAP Kinase via FieldScreen Virtual Screening. J. Med. Chem., 52, 4200-4209 (2009).

24. Rostom, S.A.F., El-Ashmawy, I.M., el Razik, H.A., Badr, M.H., Ashour, H.M.A., Design and synthesis of some thiazolyl and thiadiazolyl derivatives of antipyrine as potential non-acidic anti-inflammatory, analgesic and antimicrobial agents. Bioorg. Med. Chem., 17, 882-895, (2009)
25. Yao, M., Lam, E.C., Kelly, C.R., Zhou, W., Wolfe, M.M., Cyclooxygenase-2 selective inhibition with NS-398 suppresses proliferation and invasiveness and delays liver metastasis in colorectal cancer. Br. J. Cancer, 90, 712-719, (2004).

26. Gasparini, L., Ongini, E., Wenk, G., Non-steroidal anti-inflammatory drugs (NSAIDs) in Alzheimer's disease: old and new mechanisms of action. $J$. Neurochem., 91, 521-536 (2004).

27. Hyup Joo, Y., Kwan Kim, J., Kang, S.-H., Noh, M.-S., Ha, J.-Y., Kyu Choi, J., Min Lim, K., Hoon Lee, C., Chung, S., 2,3-Diarylbenzopyran derivatives as a novel class of selective cyclooxygenase-2 inhibitors. Bioorg. Med. Chem. Lett., 13, 413-417 (2003).

28. El-Feky, S.A.H., Abd El-Samii, Z.K., Osman, N.A., Lashine, J., Kamel, M.A., Thabet, H.K., Synthesis, molecular docking and antiinflammatory screening of novel quinoline incorporated pyrazole derivatives using the Pfitzinger reaction II. Bioorg. Chem., 58, 104116, (2015).

29. Wen, X., Wang, S.-B., Liu, D.-C., Gong, G.-H., Quan, Z.-S., Synthesis and evaluation of the antiinflammatory activity of quinoline derivatives. Med. Chem. Res., 24, 2591-2603 (2015).

30. Tassini, S., Castagnolo, D., Scalacci, N., Kissova, M., Armijos-Rivera, J.I., Giagnorio, F., Maga, G., Costantino, G., Crespan, E., Radi, M., A multicomponent pharmacophore fragmentdecoration approach to identify selective LRRK2targeting probes. Med. Chem. Commun., 7, 484494 (2016)

31. Gupta, L.K., Bansal, U., Chandra, S., Spectroscopic and physicochemical studies on nickel(II) complexes of isatin-3,2'-quinolyl-hydrazones and their adducts. Spectrochim. Acta Part A Mol. Biomol. Spectrosc., 66, 972-975 (2007).

32. Winter, C.A., Risley, E.A., Nuss, G.W., Carrageenin-induced edema in hind paw of the rat as an assay for antiiflammatory drugs. Proc. Soc. Exp. Biol. Med., 111, 544-547 (1962).

33. Haiba, M.E., Abd El-Karim, S.S., Gouhar, R.S., El-Zahar, M.I., El-Awdan, S.A., Synthesis and evaluation of anti-inflammatory and analgesic activity of some substituted thiazolyl and thaizolidinonyl tetrahydronapthalene derivatives. Med. Chem. Res., 23, 3418-3435 (2014).

34. Tzschentke, T.M., Christoph, T., Kogel, B., Schiene, K., Hennies, H.-H., Englberger, W., Haurand, M., Jahnel, U., Cremers, T.I.F.H., Friderichs, E., De Vry, J.,(-)-(1R,2R)-3-(3dimethylamino-1-ethyl-2-methyl-propyl)-phenol hydrochloride (tapentadol $\mathrm{HCl}$ ): a novel mu-opioid receptor agonist/norepinephrine reuptake inhibitor with broad-spectrum analgesic properties. $J$. Pharmacol. Exp. Ther., 323, 265-276 (2007).

35. Amer, A.M., El-Iraky, W.I., Mahgoub, S., 
Synthesis, characterization and antimicrobial activity of some novel quinoline derivatives bearing pyrazole and pyridine moieties. Egypt. J. Chem., 61(2018). DOI: 10.21608/ EJCHEM.2018.3941.1345.
36. Cunico, W., Gomes, C., Vellasco Jr., W., Chemistry and Biological Activities of 1,3-Thiazolidin-4ones. Mini. Rev. Org. Chem., 5, 336-344 (2008).

(Received 16/9/2018; accepted 29/11/2018)

\title{
تحضير وتوصيف وتقييم النشاط المضاد للالتهابات والمسكن للآلام لبعض مشتقات الثيازوليدون المتصلة بحلقة الكينولين

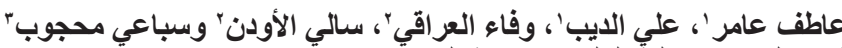

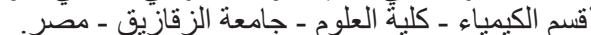

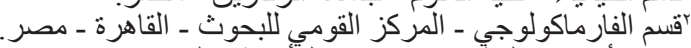

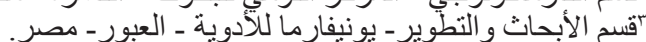

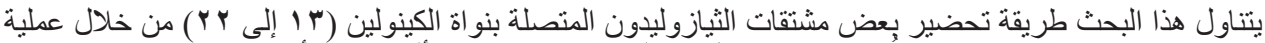

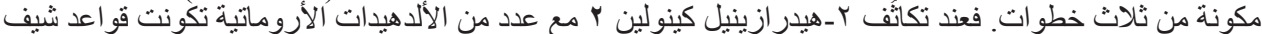

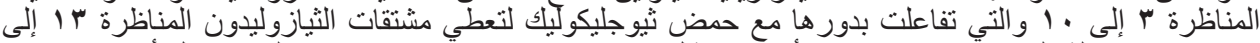

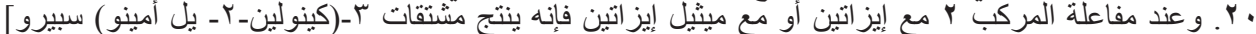

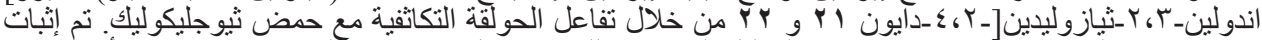

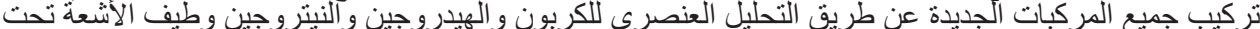

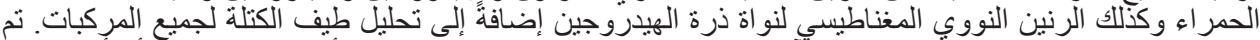

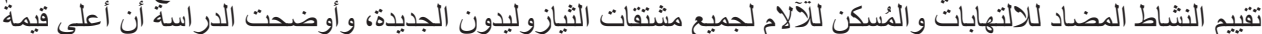

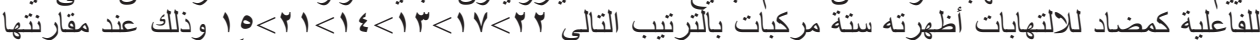

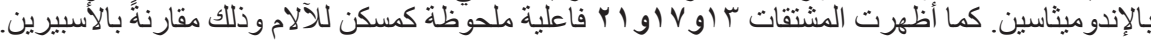

